\title{
Technology Affordances for Intersubjective Meaning-making: A Research Agenda for CSCL
}

\author{
Running Head: Technology Affordances for Intersubjective Meaning-Making \\ Alternative Running Head: A Research Agenda for CSCL \\ Daniel D. Suthers \\ Department of Information and Computer Sciences \\ University of Hawai $i$ \\ 1680 East West Road, POST 309 \\ Honolulu, HI 96822 \\ Tel: 1.808.956.3890 \\ Fax: 1.808.956.3548 \\ suthers@hawaii.edu
}

Keywords: CSCL research agenda, intersubjectivity, meaning-making, representational guidance, technology affordances

\begin{abstract}
Now well into its second decade, the field of Computer Supported Collaborative Learning (CSCL) appears healthy, while encompassing a diversity of topics of study, methodologies, and representatives of various research communities. It is an appropriate time to ask: what central questions can integrate our work into a coherent field? This paper proposes the study of technology affordances for intersubjective meaning-making as an integrating research agenda for CSCL. A brief survey of epistemologies of collaborative learning and forms of computer support for that learning characterize the field to be integrated and motivate the proposal. A hybrid of experimental, descriptive and design methodologies is proposed in support of this agenda. A working definition of intersubjective meaning-making as joint composition of interpretations of a dynamically evolving context is provided, and used to propose a framework around which dialogue between analytic approaches can take place.
\end{abstract}




\section{Introduction}

Computer Supported Collaborative Learning (CSCL) has been active for at least a decade since the 1995 conference in Bloomington, and is entering its second decade with the founding of a journal dedicated to the field. The primary purpose of this paper is to offer a research agenda for this second decade; an agenda that is one among many but is proposed as the most paradigmatic for CSCL in terms of the problems that the field now needs to address and is perhaps most uniquely ready to address. Koschmann (2002) has characterized CSCL as "a field centrally concerned with meaning and practices of meaning-making in the context of joint activity and the ways in which these practices are mediated through designed artifacts." The proposed agenda accepts but elaborates on Koschmann's definition. This paper is organized according to constituents of the definition: collaborative learning as meaning-making, approaches to mediation through designed artifacts, and methodologies for the study of these two facets of CSCL. Although the range of current theory and practice within CSCL is discussed, the argument is analytic rather than empirical, making a case for what should be the thematic focus of CSCL based on identification of those problems in the nexus of computer mediation and collaborative learning that are our special concern.

\section{CL: Learning and Meaning-making}

What is the central phenomenon of interest for CSCL? Since "computer supported" is an adjunct to "collaborative learning," let us begin by looking more closely at what we mean by the latter.

\section{Epistemologies for Collaborative Learning}

Any CSCL research agenda will be based on assumptions, implicit or explicit, concerning what it means to learn in collaborative settings. This section identifies epistemologies common in CSCL in order to understand the range of phenomena we are trying to support and to prepare for subsequent discussion. For purposes of brief exposition, the epistemologies will be presented in terms of their most distinguishing commitments, so are necessarily oversimplified. Broadly speaking, there are two kinds of accounts. In individual epistemologies, the individual is the learning agent, who may benefit from the collaborative situation. In intersubjective epistemologies, the group is the learning agent, within which individual participation may change. Between these extremes, one can postulate that learning is a group activity that results in individual changes, which we also call "learning."

Although not an epistemology of collaborative learning, constructivism (Piaget, 1976; von Glaserfeld, 1995) is frequently cited as a motivating theory in our literature and underlies some collaborative epistemologies, so is a useful starting point for the discussion. A constructivist epistemology emphasizes the agency of the learner in the learning process. Learning can only happen through the learner's efforts at meaning-making (making sense of the world), although a mentor might arrange for the learner to have challenging experiences in order to accelerate the change process. Computer support motivated by this epistemology includes simulations and "microworlds" (Rieber, 2004). All knowledge is acquired by being constructed by the learner; therefore from the standpoint of the learner, learning necessarily means constructing new 
knowledge. CSCL researchers rarely take this view to its solipsistic extreme. Instead, constructivism takes the form of "collaborative knowledge construction" (Stahl, 2000), implying an interactional constructivist epistemology. This brings us to the question of how interaction between people leads to learning.

We begin with individual epistemologies, in which the individual is the unit and agent of learning. Since we are concerned with collaborative learning, we focus on such learning that takes place "in the context of joint activity" (Koschmann, 2002; emphasis added). In an individual epistemology, collaboration provides the conditions and support for learning, but is not intrinsic to the learning itself. A social-as-context view might maintain that learning remains fundamentally a process within individual minds, yet this process can be enhanced through contacts with other minds. Cognitive dissonance theory (Festinger, 1957) and socio-cognitive conflict theory (Doise \& Mugny, 1984) can be read this way.

A knowledge-communication epistemology (Wenger, 1987) is common in the CSCL literature (e.g., Bromme, Hesse \& Spada, 2005). Knowledge communication is "the ability to cause and/or support the acquisition of one's knowledge by someone else, via a restricted set of communication operations" (Wenger, 1987, p. 7). Research conducted under this epistemology examines how to more effectively present knowledge in some medium, or how to otherwise generate or facilitate communications that "cause and/or support" the desired acquisition of knowledge. Although work that takes a knowledge-transfer view of knowledge communication continues to be published, the trend within the knowledge communication tradition is towards more constructivist and more interactional views.

Many CSCL authors (e.g., Baker, Hansen, Joiner \& Traum. 1999; Rummel \& Spada, 2005; van Der Pol, Admiraal \& Simons, 2003) build their interactionalism on the metaphor of "common ground" from Clark's contribution theory (Clark \& Brennan, 1991). Pfister (2005) proposes that adding knowledge to common ground "is the gist of cooperative learning: going from unshared to shared information." This conception of "cooperative learning" has its merits. It attributes learning to group interaction rather than to a unidirectional transfer of information between individuals. It relies on an influential model of communication that bridges psycholinguistic and social perspectives, and thereby offers CSCL a substantial research literature to draw upon. Yet, in focusing on the sharing of information (presumably that was formerly held by a subset of the participants), it does not explain how knowledge that did not predate the communication is jointly constructed within the communication process. See also Koschmann \& LeBaron (2003) for a critique of the concept of "common ground" as a "place with no place" that is only an approximation to contingently changing interpretations.

A more radically interactional epistemology, which for now will be called intersubjective learning, goes beyond an information sharing conception of collaborative learning in two ways. First, interpretations can be jointly created through interaction in addition to being formed by individuals before they are offered to the group. Cognitive activities underlying learning can be distributed across individuals and information artifacts through and with which they interact (Hollan, Hutchins \& Kirsch, 2002). In the most extreme version of this epistemology, learning is not only accomplished through the interactions of the participants, but also consists of those interactions (Koschmann et al., 2005). (This concept of learning as activity will be discussed later.) Second, intersubjectivity is to be understood in a participatory sense: it is a simultaneous 
process of mutual constitution that may involve disagreement as well as agreement about shared information (Matusov, 1996) within a "polyphonic nonharmonious concert characterized by synchronic movements, as well as by distinct, conflicting, and dissonant voices" (Smolka, De Goes, \& Pina, 1995). An intersubjective epistemology is distinguished from common ground by assuming a participatory process within which beliefs are enacted (and in this sense are shared from the outset) without necessarily being mutually accepted.

In addition to intersubjectivity on the interpersonal level, we find within CSCL intersubjective epistemologies that address learning at the community level. A participatory epistemology conceives of learning as a process of "legitimate peripheral participation" in the practices of a community (Lave \& Wenger, 1991). It is possible to read participatory accounts from an individual epistemological perspective: one becomes a member of a community by acquiring that community's cultural practices and world-view through apprenticeship. A related concept is that of internalization: developmental learning through social interaction can be understood as the internalization of interpersonal processes as intrapersonal processes (Vygotsky, 1978). However, more radical participatory epistemologies dispense with notions of acquisition or internalization and treat learning as participation (Rogoff, 1995). In this view, "learning is an integral part of generative social practice in the lived-in world" (Lave \& Wenger, 1991, p. 35) —a process that constructs personal identity, entwining individual learning with group practices that themselves can change. Although social systems are organized to replicate themselves, they can "learn" when local innovations undertaken in response to internal tensions and external disturbances redistribute activity across the system (Engeström, 2001). The new practices can be reflected in concomitant creation of novel artifacts that support and help to replicate these practices (Wartofsky, 1979).

Another community level epistemology is knowledge building, which should not be confused with the linguistically similar knowledge construction. Knowledge building is a collective version of Scardamalia and Bereiter's (1991) intentional learning. The difference is described by the Institute for Knowledge Innovation and Technology (http://ikit.org/kb.html, accessed August 31, 2004) as follows:

To understand knowledge building it is essential to distinguish learning- "the process through which the cultural capital of a society is made available to successive generations" from knowledge building - the deliberate effort to increase the cultural capital.

Scardamalia and Bereiter have worked extensively within primary school classrooms, some of which they describe as instances of "knowledge building communities." Whether knowledge is "new" is relative to the cultural capital of the community undertaking the activity, such as the knowledge available to the children in a primary school class. The essential difference between knowledge building and other forms of learning is that members of a knowledge building community expand the boundaries of their knowledge through their own collective agency by periodically reflecting on the limits of their understanding and choosing actions that address these limitations.

For simplicity, the remainder of this paper will use collaborative learning to encompass all socially contextualized forms of learning, although it should be noted that a distinction between cooperative learning as parallel coordinated activity and collaborative learning as an effort to 
maintain a joint conception is made in the literature (Dillenbourg, 1999; Roschelle \& Teasley, 1995). The other phrases are layered in the following manner: knowledge construction recognizes that individuals create meaning for themselves rather than just receiving it preformed from others; collaborative knowledge construction more specifically locates this meaning-making in a group context; intersubjective learning further specifies that the process of meaning-making is itself constituted of social interactions; and knowledge building requires that this group-based meaningmaking is being done intentionally.

\section{The Case for Studying Intersubjective Learning}

Koschmann's definition of CSCL as being concerned with the "practices of meaning-making in the context of joint activity" can be understood under many of the epistemologies previously discussed. Like the Hindu parable in which several blind men feel an elephant and each describe it differently, all are describing some aspect of the truth: learning happens in many ways. However, the question we face is how to most productively focus our research efforts: which aspect of the elephant do we now most need to understand?

The first major claim of this paper is that we most need to understand those processes of learning highlighted by intersubjective epistemologies, at both the interpersonal and community levels.

Intersubjective learning is an appropriate topic for CSCL because it is more uniquely suited to a field that conceives of itself as being concerned with collaborative learning than the other epistemologies. There has been substantial work on how the cognitive processes of participants are influenced by social interaction, and others will continue this work. The study of individual learning that is merely stimulated by a social context does not distinguish CSCL as strongly from other fields that study learning.

The study of intersubjective learning is interesting because it gives rise to questions that are among the most challenging facing any social-behavioral science, and even touches upon our nature as conscious beings. Do cognitive phenomena exist transpersonally? How is it possible for learning, usually conceived of as a cognitive function, to be distributed across people and artifacts (Salomon, 1993)? Can we understand knowledge as accomplished practice rather than as a substance or even predisposition? Yet we need not leave individual learning behind. In support of this research agenda, cognitivists can ask: What is the relationship of the change process we call "individual learning" to that individual's participation in socially accomplished learning?

The study of intersubjective learning is timely because the composition of the CSCL community is becoming increasingly well equipped to address this topic. We find among those who count themselves as members of the CSCL community people who are accomplished in various relevant disciplines and research traditions.

Finally, a call for the study of intersubjective learning is needed because it is currently not prominent as a topic of study in our field: it is surprisingly difficult to find research publications within CSCL that directly address this epistemology. (Exceptions will be noted shortly.) Even where process data is examined in detail, the analysis typically counts features that are essentially proxies for interactive accomplishment of learning (e.g., number of utterances of a 
given type) rather than exposing collaborative knowledge construction in action. The author need go no further than his own work to illustrate this point (e.g., Suthers \& Hundhausen, 2003).

\section{Learning as a Scientific Concept}

The foregoing sections surveyed a variety of accounts of collaborative learning, and concluded that while all provide some insight into learning, CSCL needs to study the intersubjective processes of learning. Following Garfinkel, Koschmann et al. (2005) argue for the study of "member's methods" of meaning-making: "how participants ... actually go about doing learning" (emphasis in original). Yet, learning was never defined. Various theories about how learning happens in group settings were discussed, but these are theories to be tested, not definitions. By what definition can we recognize that participants are "doing" learning?

The agenda outlined in this paper is deliberately designed to avoid depending on a particular definition of learning. Learning takes place within a huge diversity of activities and situations: learning is ubiquitous. Any attempt to write a single definition that covers this diversity would risk producing a concept too undiscriminating to be a productive basis for a research program, while more discriminating definitions might exclude potentially productive lines of work. The strategy taken in this paper is to integrate the field of CSCL by providing a basis for dialogue between researchers following multiple conceptions of learning and methodological traditions - a basis to be developed in this and later sections. Yet, some comments on what would count as a suitable definition of "learning" and the role of that concept in analysis may help to motivate the proposal.

If we are going to study how people go about doing learning in practice, then in order to avoid circularity in the research agenda we need an operational definition of learning that allows learning to be identified without presupposing that a particular kind of practice constitutes learning. The definition of learning taken at the outset cannot be written in terms of properties of the episodes of practice to be studied. (In contrast, an empirically derived account of learning should specify properties of practice related to learning, but this account is a product of the research program, not a definition that enables the program to be undertaken.) Therefore, a scientifically useful definition of learning is forced "outside" the episode, as it were, and must take the form of a post-hoc judgment about consequences of the episode. Various definitions of learning already in use meet this requirement, including learning as (1) gains from pre-test to post-test scores, (2) transfer of problem solving success to similar tasks, (3) an individual's attribution of an experience as having been valuable, and (4) a community's acceptance of a new member. From the standpoint of the criterion just expressed, any of these definitions are acceptable for a CSCL research agenda. All of these definitions have the property that some community makes a judgment about the consequences of an activity. No commitment to what form the post-hoc judgment takes or who makes that judgment is necessary to continue the following argument.

As a consequence of both the ubiquity of learning in diverse activities and situations and of the need for a definition of learning to be independent of that which the research program seeks to uncover, "learning" is not a concept that can be productively applied to an analysis of interaction that seeks to understand how learning is accomplished. It is a category mistake to set out to study "how people go about doing learning" in any sense that tries to interpret the actions as learning actions. We cannot say, "That was a learning act." We can say, "That act is more likely 
to lead to a particular learning accomplishment," but this is an empirically grounded description of contingencies, not a direct identification of learning itself.

\section{Intersubjective Meaning-making}

In order to understand learning, we must examine what participants are doing when they engage in an activity that leads to learning. In many of the situations from which learning can result, participants may not be engaged in an intentional effort to learn, but rather are trying to make sense of a situation (Dervin, 2003). They do so at multiple levels: solving a problem, maintaining interpersonal relationships, and/or affirming their identity in a community (Bronkart, 1995). A common denominator is the attempt to make a situation meaningful. The second major claim of this paper asks that our analysis of activity stay true to this common denominator of meaning-making:

To study the accomplishment (a post-hoc judgment) of intersubjective learning we must necessarily study the practices (the activity itself) of intersubjective meaning-making: how people in groups make sense of situations and of each other.

As previously noted, few studies published in the CSCL literature have addressed intersubjective meaning-making directly. Exceptions include Koschmann et al. (2003), Koschmann et al. (2005), Roschelle (1994), and Stahl (2004). Koschmann's work has generally focused on participants' methods of problematization: identifying a situation as problematic and requiring further analysis, possibly leading to a change of conception. Further work should identify methods for resolving the problematized issue. These will include methods for argumentation and negotiating meaning (Baker, 2003).

This author's own analytic stance is that meaning-making is accomplished (and evidenced) by the composition of interpretations of a dynamically changing context. Interpretations are enacted in human cognitive and social activity. Interpretation can be understood in terms of the participation/reification duality (Wenger, 1998). An interpretation takes a reification as having a given significance for ongoing participation, thus in effect forming a new reification. Interpretation functions as much on moment-to-moment ephemeral reifications such as thoughts, utterances, facial expressions, and gestures as on persistent inscriptions and artifacts. An act of interpretation may take the form of predications, commentary, restatements, or expressions of attitude (for example), expressed verbally, gesturally, or through manipulations of representations, and may also be "re-presented" when participants invoke inscriptions in the medium as evoking such interpretations. The perceptual environment and accumulated history of interpretations provides a rich context that participants may selectively choose to further interpret. "Composition" is used in analogy to the mathematical concept of composition of functions in order to highlight that interpretations act upon the images of previous interpretations. Intersubjective meaning-making takes place when multiple participants contribute to a composition of inter-related interpretations. In other words, the joint composition of interpretations is the gist of intersubjective meaning-making. This conception provides an alternative to "going from unshared to shared information" as the gist of cooperative learning. No commitment to mutual beliefs residing in some Platonic realm is necessary: the physical and historical context available to participants is the field upon which intersubjectivity plays. 


\section{Clarifications and Implications}

The claim that it is now time for CSCL to focus on practices of intersubjective meaning-making is offered as a strategic choice. Others may choose to prioritize different directions for the field. On the other hand, the claim that it is inappropriate to use "learning" as an analytic concept in understanding "how people go about doing learning" is offered as an absolute claim, independently of the foregoing strategic choice. The claim that members' methods of intersubjective meaning-making is the appropriate analytic concept is more agnostic concerning epistemology than it might seem. Learning can still be conceived of as individual internalization that results from a social activity of meaning-making (including Vygotskian internalization of the social activity of meaning-making itself). One can equally take the opposing view that “... orienting our inquiry by focusing on how people participate in sociocultural activity and how they change their participation demystifies the processes of learning and development" by eliminating the need to search for "the nature of internalization as a conduit" (Rogoff, 1995). In advocating an intersubjective stance as a strategic choice, this paper does not reject the cognitive agenda, but rather asks that all paradigms focus on intersubjective meaning-making as a shared object of contemplation - a "boundary object" (Star, 1990) that will give the field the basis for coherence through dialogue between traditions.

Practices of intersubjective meaning-making are found in potentially any and every kind of joint human activity. One might object that the proposal requires that we attempt to understand all of human collaborative activity, and CSCL would have lost its focus. The objection is partially sustained: CSCL is indeed potentially concerned with all of human collaborative activity (learning as a consequence of activity is always a possibility), but there is still a focus to CSCL's learning science agenda. The focus is not defined by limiting consideration to certain kinds of activity (e.g., activities in institutionally-sanctioned learning settings such as "schooling," or more generally situations in which there is the intention to learn or to teach). Rather, the focus is defined by what aspect of human collaborative activity we examine and try to make sense of: intersubjective meaning-making.

This view of the scope of CSCL elevates the potential impact of the field. CSCL need not be conceived of as merely a subfield of a subfield (e.g., a specialization of collaborative learning within educational psychology). If we succeed in shedding light on intersubjective meaningmaking, it can inform many fields of inquiry. Because of the potential for misunderstandings, it should be emphasized that the author is strongly supportive of the study of learning. The call to replace learning with meaning-making as an analytic concept in understanding learning is done out of necessity. If we are to serve learning well, we must grapple with intersubjective meaningmaking, and in so doing will be achieving something larger as well, whether we wish to or not. Therefore we might as well accept this larger agenda and celebrate the relevance and longevity of our field that it portends.

\section{CS: Computer "Support" or Mediation}

Let us now add computers to the mix. In what ways can we bring technology to bear on the problem of supporting collaborative learning as it is variously conceived, and in particular intersubjective meaning-making? This section identifies two distinct ways in which technology is 
applied to support collaborative learning - as medium and as constraint - and then proposes a synthesis. (See also Hansen, Dirckinck-Holmfeld, Lewis \& Rugelj (1999) for a synthesis of "compensating" and "facilitating," and Jerman, Soller \& Lesgold (2004) for "structuring" and "regulating.") The prior discussion is relevant because our choice of an epistemology of collaborative learning can affect how we approach the design of computer mediation and what questions we ask in our research. For example, under a knowledge-communication model, we might think about the information technologies we are designing as communication channels, focusing on the ease with which one can move information between participants. Under an intersubjective meaning-making model, we might design information technologies as forums within which new ideas can be jointly formed - or discovered - and evaluated. However, it is also possible to support collaboration without making any particular commitment to a theory of collaborative learning. We first consider an approach that minimizes its epistemological commitment.

\section{Technology as Interaction Medium}

Some approaches to computer support treat technology as a communication channel in a manner that is neutral to learning. Computer support enables interaction (and perhaps collaboration); learning is left as incidental or up to the participants to achieve.

People often resort to computer-mediated communication (CMC) as a substitute for face-toface (FTF) interaction in order to make interaction possible between people at different locations (synchronous distance interaction) or at different times (asynchronous interaction). It is not surprising that FTF interaction would then be taken as the standard against which CMC is evaluated (Olson \& Olson, 2000). Research in this tradition tries to improve the bandwidth and multimodality of CMC technology and fine-tune its design to match the characteristics of FTF. For example, gaze and gesture are demonstrably vital cues in FTF interaction, so some researchers study how to arrange cameras such that the remote image of a person gives a more accurate indication of where they are looking or pointing (e.g., Kato et al., 2001). Without denying that face-to-face interaction has great value, it is instructive to consider why technologyoriented research in CSCL should not be conceived of as merely seeking online replication of the multimodality of FTF learning. Four reasons are offered.

First, CSCL does not necessarily replace FTF interaction. Computational artifacts can also augment spoken and gestural communication between co-present collaborators (Roschelle, 1994; Suthers \& Hundhausen, 2003), and be embedded in classrooms where much of the interaction is FTF (Lingnau, Hoppe \& Mannhaupt, 2003; Scardamalia \& Bereiter, 1991; Toth, Suthers \& Lesgold, 2002).

Second, although further progress can be made, ultimately the goal of replicating FTF interaction online may not be achievable. "Distance matters" (Olson \& Olson, 2000) in many subtle ways when collaborating through technology. Even with extremely high bandwidth communication in multiple modalities, some advantages of spatial co-location will be difficult to replicate online, such as access to implicit contextual information, unconstrained gaze and gesture as cues for identifying deictic referents, and the use of interpersonal space to coordinate action.

Third, it is not sufficient for CSCL to merely replicate FTF interaction. As Pfister (2005) puts it "even if virtual reality is achieved ... genuine learning discourse is not supported. It is 
completely up to the participants ... how to structure the learning process." Rather than leaving efficient learning up to the learners, CSCL has an obligation to design technology that supports effective collaborative learning. In order to do so, some commitment to an epistemology is necessary.

Fourth, CSCL can explore the advantages of going "beyond being there" (Hollan \& Stornetta, 1992): ways in which CMC is actually better than FTF. An obvious example is that CMC "turns communication into substance" (Dillenbourg, 2005), providing additional resources for learning. The record of contributions and shared representations that are manipulated during communication provide a shared persistent information base that enables the community of collaborators to reflect and act on its own state of understanding-to reinterpret, find connections between, refine and expand information and ideas explored over time.

Research that focuses primarily on supporting collaboration through CMC but does not necessarily directly address issues of learning might be considered peripheral to CSCL. However, under the proposed agenda, understanding the affordances technology offers for intersubjective meaning-making is as foundational to CSCL as understanding learning. (Although "affordances" originated with Gibson (1977), in this paper, the term is used in Norman's (1999) sense of "perceived affordances," widely adopted in the human-computer interaction literature.) Much further work is needed to answer questions such as: What strategies do people use to manage collaboration and meaning-making via artifact-mediation? How are the affordances of various media (including but not limited to information technologies) appropriated to carry out these strategies? How then can we design information technologies to provide functionally equivalent affordances with the most natural match to the observed strategies? (Dwyer \& Suthers, 2005).

\section{Technology as Constraint and Guide}

Computational technologies, as well as other information technologies such as paper based instructional materials, are often applied to education as means to limit the options available to learners. Although it sounds negative, this is sometimes a useful strategy, for two major reasons: reducing socio-cognitive load and implementing a learning agenda.

Properly applied, constraints on activity can resolve a paradox of collaborative learning. Collaboration imposes an additional task on the learners: in addition to choosing actions within the problem domain and evaluating the consequences of those actions, they must also manage interpersonal relations and group functioning (Whitworth, Gallupe \& McQueen, 2000). Learning may be reduced if cognitive resources are diverted from the primary task (Sweller, van Merriënboer, \& Paas, 1998). However, if learners can help each other with different parts of the problem, collaboration can reduce task load. Furthermore, collaboration can increase learning effectiveness through activities that are more difficult to do alone, such as argumentation, explanation and reflection (Andriessen, Baker \& Suthers, 2003; Slavin, 1995). To resolve this paradox, instructional technology is often designed to structure part of the activity, "offloading" work onto the technology so that learners can focus their cognitive and social resources on other relevant aspects of the learning activity. The technology support can take different forms, such as full automatization of the offloaded task, constraining actions to reduce the need to make decisions while executing the task, or non-mandatory guides such as coaching agents or representational guidance. Whatever form it takes, this support might be subsequently removed 
(the "scaffolding" "fades" in this mixed metaphor) as learners internalize the guidance it provided. This strategy is called a reduction of sociocognitive load strategy, expanding on Sweller, et al.'s (1998) concept of cognitive load, because the strategy addresses the capacity of the group, not just individuals, to manage multiple task demands at once. Important research topics include determining what to scaffold (Weinberger, Reiserer, Ertl, Fischer \& Mandl, 2005), comparing the effectiveness of different forms of scaffolding (Rummel \& Spada, 2005), optimizing fading strategies, and exploring whether the answers to these questions generalize in any predictable ways across task domains.

Technology constraints can also be used to implement a learning agenda. Analysis of the learning task may reveal prerequisites, or uncover difficulties that are best left for after fundamental skills are learned. Then, guidance is applied via any of the methods previously listed (automatization, interface constraints, coaches, representational guidance) to ensure that skills are acquired or new challenges are taken on in an optimal order. Choices of what parts of the task to "scaffold" and how to sequence "fading" can be effective ways to implement a learning agenda. Similarly, constraints can be used to enforce a collaboration protocol, perhaps one based on an epistemological commitment as to what constitutes learning through collaboration (e.g., Jermann \& Dillenbourg, 2003; Weinberger et al., 2005) For example, some researchers have identified collections of conversational moves that they believe are necessary for an effective learning dialogue, and implemented these moves as mandatory sentence openers in a communication interface (e.g., Baker \& Lund, 1997; Robertson, Good \& Pain, 1998).

Some ways in which technology can be used to guide and support collaborative learning are not intrinsic to the technology itself. For example, consider scripting and role-playing. We might prompt participants to go through phases of collaboration, or provide protocols for making and evaluating proposals. These interventions could just as well be done with paper, or even verbal instructions. There are clear advantages to using computational technology, such as support for distance interaction and automated prompting, but the primary variable being studied is not itself a property of computational technology (see also Dillenbourg, 2002).

From the point of view of theories that claim to be able to prescribe activities for learners, technology-as-constraint has great value. Indeed, domain-specific (Shulman, 1987) and even problem-specific (Anderson, Corbett, Koedinger \& Pelletier, 1995) guidance is seen as critical to learning success. However, domain-specific guidance is more of a problem for instructional design than one specific to the unique concerns of CSCL. Also, the use of technology as guide and constraint risks inflexibility, and may be inappropriate for learner-driven epistemologies such as intersubjective meaning-making and knowledge building. Under these epistemologies, we do not want to limit the potential meanings that can be expressed or trajectories of joint action through which a group approaches a problem. Rather, we want to uncover and exploit affordances to make these easier.

\section{Technology Affordances for Intersubjective Meaning-making}

In order to serve the intersubjective meaning-making agenda, a selective synthesis of the two uses of technology mediation just discussed is needed. Richer communication media are needed, particularly with respect to supporting the indexical nature of human communication (Nunberg, 1993). Guidance for a learning agenda is needed for both discipline-specific practices and learning 
trajectories and for processes of intersubjective meaning-making, but without limiting creativity by excessively rigid scripting of action. In order to achieve advancements in these forms of support, we need to better understand "the ways in which these practices [meaning-making in the context of joint activity] are mediated through designed artifacts" (the second half of Koschmann's definition of CSCL). The third major claim of this paper follows:

The technology side of the CSCL agenda should focus on the design and study of fundamentally social technologies that are informed by the affordances and limitations of those technologies for mediating intersubjective meaning-making.

CSCL systems should be fundamentally social because interactional and especially intersubjective epistemologies of learning require this. To be fundamentally social means that the technology should be designed specifically to mediate and encourage acts of intersubjective meaning-making. To be informed by the affordances and limitations of a technology means that the design attempts to leverage the unique opportunities provided by the technology rather than replicating support for learning that could be done through other means, or (worse) trying to force the technology to be something for which it is not well suited.

The research agenda surrounding technology affordances for intersubjectivity is rich. We first need to understand what collaborative strategies people use when communicating via information artifacts of all types. Human communication and the use of representational resources in its service are flexible: we cannot specify meanings or communicative functions for those resources in advance. Instead, CSCL research should identify how collaborators appropriate perceived affordances of media (Norman, 1999), and explore how notational properties (e.g., Blackwell \& Green, 2003) of media influence the course of collaboration. Interactional strategies that recur across a variety of media are likely to be essential (Dwyer \& Suthers, 2005). People will try to find a way to apply them regardless of how viscous the medium is with respect to those strategies. Our job as designers is to find more natural mappings, offering collections of affordances that support participants' strategies while providing flexible forms of guidance (see also Kirschner, Martens \& Strijbos, 2004). The remainder of this section discusses some unique opportunities computational technology provides for intersubjective meaning-making, suggesting specific lines of investigation for the proposed research agenda.

(Im)mutable Mobiles. As a notational medium, the computational medium is reconfigurable and replicable. It is easy to manipulate digital objects and to replicate actions and objects elsewhere: one can bridge time and space. The mobility of digital inscriptions - both mutable and immutable - provides opportunities for recruitment of partners in the sense-making process (Latour, 1990) and supports continued engagement in that process. How can we exploit this property of technology for its potential to make new social alignments and their interactions possible?

Negotiation Potentials. Any medium offers certain potentials for action. To the extent that inscriptions within the medium are socially shared (e.g., representations of problem solutions in a synchronized workspace), participants may feel an obligation to obtain agreement on modifications to those inscriptions. The potentials for action offered by the medium can therefore guide interactions towards ideas associated with the afforded actions (Suthers \& Hundhausen, 2003). An analysis can begin by asking: what constructive actions does the medium 
enable? Which possibilities for action are most salient (i.e., are perceived affordances)? What decisions must be made to choose and carry out one of these actions? If participants negotiate these decisions, will their interactions be productive for learning according to the epistemology guiding the design? Design can apply this analysis in reverse: if we would like users of our technology medium to focus on particular aspects of a problem, how can the medium be designed to prompt for actions that require negotiation of these aspects?

Referential Resource. Jointly constructed representations become imbued with meanings for the participants by virtue of having been produced through a process of negotiation. These representational constituents then enable reference to prior interpretations with deictic reference (through gesture or language), or by direct manipulation (Suthers, Girardeau, \& Hundhausen, 2003). In this manner, collaboratively constructed external representations facilitate subsequent negotiations; increasing the conceptual complexity that can be handled in group interactions and facilitating elaboration on previous conceptions. The expressive and indexical affordances of a medium will affect its value as a referential resource. Therefore we might consider how to make salient that which we would like our technology users to elaborate on and relate to new information or ideas. What interpretations (e.g., ideas or elements of the argumentation or problem solution) do participants tend to assign to representational proxies? How can the indexicality necessary for subsequent interpretive acts be accomplished in our technologymediated settings?

Similarly, disciplinary representations such as models, simulations and visualizations also offer negotiation potentials and serve as resources for conversation. Rather than being vehicles for communicating expert knowledge, such representations become objects about which learners engage in sense-making conversations (Roschelle, 1994) and can be designed to lead to productive conversation.

Integration. Inscriptions in the computational medium can be persistent. A record of activity and its products can be kept, replayed, and modified. This property can be selectively exploited to leverage prior activity as a learning resource, enabling compositions of interpretations that transcend distribution across time and individuals. We should explore how a persistent record of interaction and collaboration can serve as a resource for intersubjective meaning-making through reflection on prior activity. How can representational artifacts be designed to foster appropriate awareness of prior conceptions and the means to reference these in subsequent interactions so that they may be integrated with new information and ideas?

Trajectories of Participation. What are the social affordances of technologies for patterns of participation over larger spans of time and collections of actors? In what ways and at what scales can multiple transformations of representations distributed across individuals and time be collectively understood as a joint meaning-making process? Can we encourage productive entanglement of multiple individual trajectories of participation by selectively making their reifications salient and hence available for subsequent interpretation by others?

Adaptiveness. A computational medium can analyze workspace state and interaction sequences, and reconfigure itself or generate prompts according to features of either. We should explore the potential of conditional dynamism as an influence on the course of intersubjective processes. We need not anthropomorphize the medium to take advantage of its ability to prompt, analyze and selectively respond. 
Reflector of Subjectivity. Computational media can be designed to foster group awareness (e.g., Kreijns \& Kirschner, 2004). The mere awareness that others are present and will evaluate one's actions may influence one's choice of actions (Erickson \& Kellog, 2000). Information about the attentional status of group members and their attitudes towards previously proposed ideas may influence the actions of individuals in the group. Visualizations of conflict or agreement between members may lead to further argumentation or reaching of consensus (Jermann \& Dillenbourg, 2003). Technology can enhance intersubjective meaning-making by projecting representations of self into a social representation (Kaput \& Hegedus, 2002) or embedding the physical self in a social simulation (Colella, 2002). In what specific ways can we design technology to mediate intersubjectivity by reflecting activity, subjectivity, and identity?

All of these questions of how the properties of technology can not only enable but also be appropriated for intersubjective learning are concerned with social technology affordances. The study of technology affordances should be undertaken with constant reference to the activity to be supported -- intersubjective meaning-making and its consequences for learning.

\section{Methodological Considerations}

What methodological approach is most suited for the proposed study of technology mediation of intersubjective meaning-making? This section first considers the major methodological traditions of CSCL and the granularities at which they may be applied, and then offers a framework for multivocal analysis that is motivated by the definition of intersubjective meaning-making previously advanced.

\section{Methodological Diversity and Synthesis}

CSCL can presently be characterized as consisting of three methodological traditions: iterative design, experimental, and descriptive.

The iterative design tradition is exemplified by Barab \& Squire (2004), Fischer \& Ostwald (2005), Guzdial et al. (1997), and Lingnau, et al. (2003). Design-oriented researchers continuously improve artifacts intended to mediate learning and collaboration, driven by the dialectic between theory and informal observations and engaging stakeholders in the process. Their research might best be understood as "quisitive" (Goldman, Crosby, Swan \& Shea, 2004) rather than qualitative versus quantitative. Exploring design is a valuable component of the overall CSCL portfolio of research strategies. We are trying to uncover the potential affordances of information technologies, so need to explore the "space" of possible designs, pushing into new areas and identifying promising features. However, iterative design alone lacks methods for predicting the implications of its design choices. We look to another tradition for the establishment of dependencies between interventions and outcomes.

Many empirical studies follow the dominant experimental paradigm that compares an intervention to a control condition in terms of one or more variables (e.g., Baker \& Lund, 1997; Rummel \& Spada, 2005; Suthers \& Hundhausen, 2003; Van Der Pol et al., 2003; Weinberger et al., 2005). Data analysis in most of these studies is undertaken by "coding and counting:" interactions are categorized and learning outcomes measured, and group means are compared through statistical methods in order to draw generalizable conclusions about the effects of the 
manipulated variables on aggregate (average) group behavior. Typical studies do not directly analyze the accomplishment of intersubjective meaning-making. Such an analysis must examine the structure of specific cases of interaction rather than categorize and aggregate single contributions. Therefore, experimental studies have been criticized for missing the point, although this limitation is not intrinsic to the experimental approach, but rather to the methods of analysis used. Another critique concerns the weak external (ecological) validity of studies based on contrived situations.

Descriptive research addresses these concerns through methods that are more suited for understanding authentic practice through case studies. These include Conversation Analysis (Sacks, Schegloff, \& Jefferson, 1974), Interaction Analysis (Jordan \& Henderson, 1995), Grounded Theory (Glaser \& Strauss, 1967), and Narrative Analysis (Hermann, 2003). Descriptive methods are exemplified in CSCL by Baker (2003), Roschelle (1994), Koschmann et al. (2003), Koschmann, et al. (2005), and Yukawa (2005). Typically, video or transcripts of activity in "natural" settings are studied to uncover the methods by which participants accomplish learning. The approach is data-driven, seeking to discover patterns in the data rather than imposing theoretical categories. Some descriptive methods such as conversation analysis are microanalytic, examining brief episodes in great detail, but others such as narrative analysis address phenomena at a larger scale. Descriptive methodologies are well suited to existentially quantified claims (e.g., that a community sometimes engages in a given practice). Yet, as scientists and designers we would like to make predictive generalizations about the effects of design choices. Descriptive methodologies are less suited for claiming that an intervention has an effect, the province of experimental methodology.

If we focus on finding examples of how members accomplish learning, we may miss abundant examples of how they also fail to do so. Yet in order to find that something is not there, we need to have an idea of what we are looking for. A purely data-driven approach that derives but never applies theory does not complete the job. An iterative comparative approach can be applied to address this need. Common patterns found in successful learning episodes subsequently become the theoretical categories we look for elsewhere, and perhaps do not find in instances of unsuccessful collaboration. Having identified where the successful methods were not applied, we can then examine the situation to determine what contingency was missing or responsible. Care should be taken, however, to make sure that in finding case examples where the interactional accomplishment of learning as we define it is absent we do not fail to notice where something else of value to the participants is being accomplished! For example, establishment and maintenance of individual and group identity are also worthwhile accomplishments as far as the participants are concerned (Whitworth et al., 2000), and indeed are a form of learning, whether or not they are aligned with researchers' or institutionally sanctioned learning objectives.

The foregoing discussion of complementary traits suggests that we explore mixed and hybrid research methodologies, drawing upon the strengths of each (Creswell, 2003; Häkkinen, Järvelä, \& Mäkitalo, 2003; Johnson \& Onwuegbuzie, 2004). Multiple forms of mixed method research are possible. Creswell (2003) discusses various sequential and concurrent strategies. In a sequential strategy, one method is used to locate portions of the data to be analyzed by other methods. For example, traditional quantitative analyses, including coding and counting of interaction categories and measures of learning outcomes, might be used to obtain quick indicators 
of where more detailed descriptive analyses are merited, thereby focusing the time-consuming work. Conversely, descriptive analyses can be used to identify the affordances of designed artifacts that seem to be correlated with effective learning episodes, thereby isolating variables that can be explored systematically in experimental designs. Concurrent triangulation strategies apply multiple methods independently of each other in order to obtain a consistency check (if they are addressing the same aspect of the phenomenon) or to obtain a richer understanding of the phenomenon from different perspectives (if they address different aspects). For example, Koschmann, Zemel \& Stahl's (2004) suggest that ethnomethodology be applied to understand practice in the context of design-based research. Concurrent nested strategies combine multiple methods into a single analysis. For example, experimental designs can compare interventions in terms of descriptive analyses of how the features of information technology influence and are appropriated for members' methods of joint meaning-making. This fusion raises the level of experimental "coding and counting" to patterns of meaning-making that are less subject to the critique of missing the point, while providing the descriptive methodology with systematically varied contexts that sanction correspondingly systematic generalizations. Such analyses are time intensive: researchers will need instrumentation of learning environments and automated visualization and querying of interaction logs as research aids. In each of these examples, the synthesis need not relegate either family of methodologies to subservient roles. For example, a conversation between the theoretical assumptions of ethnomethodology and those of design can lead to a "technomethodology" that changes the very objectives of design (Button \& Dourish, 1996).

\section{Unit of Study}

Stahl (in press) argues that small groups are the most fruitful unit of study, for two reasons. Most simply, small groups are where members' methods for intersubjective meaning-making can be observed. Groups of several members allow the full range of social interactions to play out, but are not so large for participants and researchers alike lose track of what is going on. More interestingly, small groups lie at the boundary of and mediate between individuals and a community. The knowledge building that takes place within small groups becomes "internalized by their members as individual learning and externalized in their communities as certifiable knowledge" (Stahl, in press). However, small groups should not be the only social granularity studied. For example, understanding the emergence of social and knowledge capital in a community of practice may require tracing out the evolution of relationships and the formation and spread of ideas in networks of individuals larger than the small group (Resnick, 2002; Wenger, McDermott \& Snyder, 2002). Analysis of large-scale changes in communities and organizations may lead to understanding of emergent social learning phenomena (Engeström, 2001) as well as elucidate the role of embedded groups in driving these changes. At the other extreme, Shaffer \& Clinton (2005) argue that even the interaction between an individual and technology can be understood as collaborative. 


\section{Eclectic Analysis of Uptake}

In the proposal under consideration, multiple theoretical and methodological traditions are brought to bear on the problem of understanding technology-mediated intersubjective meaningmaking. This final section proposes a framework for eclectic analysis.

Intersubjective meaning-making requires interactions between participants (interpretations of reifications of actions of another participant). Any analysis of intersubjective meaning-making, whether microanalytic or concerned with the dynamics of the community or culture evolving through time, must begin by identifying uptake acts in which one participant takes up another's contribution and does something further with it. Contributions may include attentional orientation, information, or expressions of attitude, reified as media affordances allow. Examples of uptake include "A has expressed proposition $\mathrm{P}(\alpha), \mathrm{B}$ expresses $\mathrm{Q}(\alpha)$, or $\mathrm{Q}(\mathrm{P}(\alpha))$," "A says $\mathrm{P}$ and $\mathrm{B}$ expresses (dis)agreement," "A makes object $\mathrm{O}$ available, and $\mathrm{B}$ attends to $\mathrm{O}$," "A has created object $\mathrm{O} 1$; B has changed it to O2," "A has created O1 and B has created O2; now A combines $\mathrm{O} 1$ and $\mathrm{O} 2$ in such a manner," etc.

In order to begin with a defensible starting point for analysis, we consider only uptake relations that are evidenced by the observable dependence of an act on others or their products. Inferences that require further theoretical commitments are left for subsequent analysis. In order to support analysis of both personal and group processes and how the two are intertwined, both intra- and inter-subjective uptake relations are included. The resulting collection of uptake relations may be conceived of as a directed acyclic graph (embedded in a temporally continuous process) consisting of arcs between points at which we have evidence (grounded in use of media affordances) of perceptions and/or expressions of attention, attitudes and conceptions.

Once we have identified a portion of this uptake structure, we need to recognize what the participants have accomplished through sequences or compositions of uptakes, and we need to identify the potential influence or utilization of technology affordances in this accomplishment. What do we look for in order to identify the acts of interpretation and meaning-making accomplished through the uptake? Different analytic approaches offer different answers to this question (Suthers, 2005). The uptake graph becomes a boundary object (Star, 2000) towards which theoretical and methodological discourse between these analytic approaches may be directed. We can layer interpretations on this graph, working from the physical actions and their interdependencies to inferences concerning participants' personal and intersubjective meaningmaking processes. Multiple interpretations can be juxtaposed and compared. There will always be multiple interpretations because an action can be understood simultaneously as an act on the objective world, an attempt to conform to behavioral norms, and a way of constructing one's identity in the social world (Bronckart, 1995); and participation in a community can be understood on three "planes" (Rogoff, 1995). Also, collaborative knowledge construction involves multiple processes (see figure 9-1 of Stahl, in press). An eclectic approach that "triangulates" from multiple theoretical perspectives is necessary due to the complexity of the problem we are tackling. We can draw upon various theories for insights on what counts as interpretive acts and what those acts mean for the learning of individuals and groups.

This framework was applied in an analysis of participant's manipulations of a shared workspace during synchronous online collaboration in order to determine whether and how such 
actions can be understood as accomplishing collaborative knowledge construction (Suthers, 2005). The analysis explored the potential contribution of different theoretical stances, including contribution theory, socio-cognitive theories, distributed cognition, and activity theory. There are other theories that can be applied to the process of generating researchers' interpretations of uptake relations as evidence of participants' composition of interpretations of their dynamically evolving context. The challenge is to take the step from affordances defined in terms of features of representations to the social level and make predictions of the opportunities the technology provides for discovering affinities with others, orienting attention, expressing viewpoints, exposing conflict and consensus, and supporting debate and negotiation. We have at our disposal a powerful repertoire of theories of learning and social interaction, and have not yet fully explored the analytic power of this repertoire. Incompatibilities between the fundamental worldviews of proponents of these theories do exist, but this does not prevent those of us who are open to a multivocal understanding of the phenomena we study from appropriating the insights of each theory and applying them towards achieving this understanding.

\section{Conclusions}

CSCL is a field that is establishing basic yet sometimes peripheral findings as it seeks its center. Work currently being undertaken in the field is undertaken through diverse methods, encompasses several epistemologies of collaborative learning, and leverages information technology as communication medium and as a constraining and guiding medium. However, there is an emerging awareness that we need to grapple with the central and most unique problem of CSCL: processes of intersubjective meaning-making and how technological affordances mediate or support such processes.

Research methodology in CSCL is largely trichotomized between experimental, descriptive and iterative design approaches. Although sometimes combined within a single research project, the methodologies are even then typically kept separate in companion studies or separate analyses of a single study. This situation can be productive for a little longer, as the experimentalists continue to identify variables that affect general parameters of collaborative behavior, while the ethnomethodologists identify patterns of joint activity that are essential to the meaning-making and learning we all seek to support. However, very soon CSCL needs experimentalists to study dependent variables that directly reflect the phenomenon of interest, the ethnomethodologists to look for predictive regularities in technology-mediated meaningmaking that can inform design, and the designers to generate and assess promising new technology affordances in terms of the meaning-making activities they enable. Mutual assistance is possible through sequentially and concurrently hybrid methodologies, and through computer support for our own meaning-making activities as researchers. A common focus on intersubjective meaning-making will serve to increase the dialogue between subcommunities of CSCL. A framework for analysis was offered in which inter- and intra-subjective "uptakes" grounded in observed uses of media affordances are identified, forming a graph that serves as a common starting point for multiple analyses exploring participants' personal and intersubjective meaning-making processes, and as a boundary object for discourse between the theoretical traditions that inform these analyses. This paper is offered in hopes of accelerating an impending 
shift in our field - towards the study of practices of intersubjective meaning-making and how these practices are mediated by technology affordances.

\section{Acknowledgements}

This is an expanded and revised version of a paper presented at the CSCL 2005 conference, which originated in a commentary on Bromme et al. (2005). I am in gratitude to Tim Koschmann and Gerry Stahl for comments on drafts and especially for their papers that have challenged my thinking; to Nathan Dwyer for years of stimulating discussions and for extensive comments on drafts of the present paper; to reviewers of the CSCL 2005 version for deeply insightful and detailed comments, the most challenging of which remain to be addressed; and to Vi Harada and Ravikiran Vatrapu for additional insights and commentary. I dedicate this paper to James Kaput, an inspiration to many, who requested a draft shortly before he died. This work was supported by the National Science Foundation under award 0093505. Any opinions, findings, and conclusions or recommendations expressed in this paper are those of the author and do not necessarily reflect the views of the National Science Foundation or those who have graciously offered their assistance.

\section{References}

Anderson, J. R., Corbett, A. T., Koedinger, K. R., \& Pelletier, R. (1995). Cognitive tutors: Lessons learned. The Journal of the Learning Sciences, 4 (2), 167-207.

Andriessen, J., Baker, M. \& Suthers, D. (Eds.) (2003). Arguing to Learn: Confronting Cognitions in Computer-Supported Collaborative Learning Environments. Dordrecht: Kluwer.

Baker, M. (2003). Computer-mediated argumentative interactions for the co-elaboration of scientific notations. In Andriessen, Baker \& Suthers (2003), op cit. (pp. 47-78).

Baker, M, Hansen, T., Joiner, R. \& Traum, D. (1999). The role of grounding in collaborative learning tasks. In Dillenbourg (Ed.) (1999). op cit. (pp. 31-63).

Baker, M. \& K. Lund (1997). Promoting reflective interactions in a CSCL environment. Journal of Computer Assisted Learning, 13, 175-193.

Barab, S. \& Squire, K. (2004). Design-Based Research: Putting a Stake in the Ground. Journal of the Learning Sciences, 13(1), 1-14.

Blackwell, A. \& Green, T. (2003). Notational Systems-The Cognitive Dimensions of Notations Framework. In J. M. Carroll (Ed.), HCI Models, Theories and Frameworks: Towards a Multidisiplinary Science (pp. 103-133). San Francisco, Mogan Kaufmann.

Bronckart, J. P. (1995). Theories of action, speech, natural language, and discourse. In J. V. Wertsch, P. Del Rio, \& A. Alvarez (Eds.) Sociocultural Studies of Mind. (pp. 75-91.) New York: Cambridge University Press.

Bromme, R., Hesse, F.W. \& Spada, H. (Eds.) (2005). Barriers and Biases in Computer-Mediated Knowledge Communication - and How They May Be Overcome. Dordrecht: Kluwer.

Button, G. Y. \& Dourish, P. (1996). Technomethodology: Paradoxes and Possibilities. Proc. ACM Conference on Human Factors in Computing Systems (CHI'96), April 13-18, 1996, Vancouver, BC, 19-26. New York: ACM. 
Clark, H.H. \& Brennan, S.E. (1991). Grounding in Communication. In L.B. Resnick, J.M. Levine and S.D. Teasley (Eds.), Perspectives on Socially Shared Cognition. (pp. 127-149). Hyattsville, MD: American Psychological Association.

Colella, V. (2002). Participatory simulations: Building collaborative understanding through immersive dynamic modeling. In T. Koschmann, R. Hall \& N. Miyake (Eds.), CSCL 2: Carrying Forward the Conversation. (pp. 357-391). Mahwah, NJ, Lawrence Erlbaum.

Cresswell, J. W. (2003). Research Design: Qualitative, Quantitative, and Mixed Methods Approaches. Sage Publications, 2003.

Dervin, B. (2003). Chaos, order, and sense-making: A proposed theory for information design. In B. Dervin, L. Foreman-Wernet with E. Lauterbach (Eds.) Sense-Making Methodology Reader Selected Writings of Brenda Dervin. (p. 325-340). Cresskill, NJ: Hampton Press.

Doise, W. \& Mugny, G. (1984). The Social Development of the Intellect. Oxford: Pergamon Press.

Dillenbourg, P. (Ed.) (2004). Collaborative Learning: Cognitive and Computational Approaches Amsterdam: Elsevier.

Dillenbourg, P. (1999). What do you mean by "collaborative learning"? In Dillenbourg (Ed.) (2004), op cit. (pp. 1-19).

Dillenbourg, P. (2002). Over-scripting CSCL: The risks of blending collaborative learning with instructional design. In P. A. Kirschner (Ed.), Three worlds of CSCL. Can we support CSCL. (pp. 61-91). Heerlen: Open Universiteit Nederland.

Dillenbourg, P. (2005). Designing biases that augment socio-cognitive interactions. In R. Bromme, F.W. Hesse \& H. Spada (2005), op. cit. (pp. 243-264).

Dwyer, N. \& Suthers, D. (2005). A Study of the Foundations of Artifact-Mediated Collaboration. In T. Koschmann, D. Suthers \& T.W. Chan (Eds.) Computer Supported Collaborative Learning 2005: The Next 10 Years! (pp. 135-144.) Mahwah, NJ: Lawrence Erlbaum Associates.

Engeström, Y. (2001) Expansive learning at work: Toward an activity theoretical reconceptualization. Journal of Education and Work 14(1): 133-156.

Erickson, T. \& Kellogg, W. A. (2000). Social Translucence: An approach to designing systems that support social processes. ACM Transactions on Computer-Human Interaction, 7(1), March 2000, pp. 59-83.

Fischer, G. \& Ostwald, J. (2005). Knowledge communication in design communities. In R. Bromme, F.W. Hesse \& H. Spada (Eds.), op. cit. (pp. 213-242).

Gibson, J. J. (1977). The theory of affordances. In R. Shaw \& J. Bransford (Eds.), Perceiving, Acting and Knowing (pp. 67-82). Hillsdale, NJ: Erlbaum.

Glaser, B. G., \& Strauss, A. L. (1967). The discovery of grounded theory: strategies for qualitative research. Chicago: Aldine.

Goldman, R., Crosby, M., Swan, K. \& Shea, P. (2004). Introducing Quisitive Research: Expanding qualitative methods for describing learning in ALN. In Starr Hiltz, R. \& Goldman, R. (Eds.), Learning Together Online: Research on Asynchronous Learning Networks. (pp. 103-121). Mahwah, New Jersey: LEA.

Guzdial, M., Hmelo, C., Hubscher, R., Newstetter, W., Puntambekar, S., Shabo, A., Turns, J., \& Kolodner, J. (1997). Integrating and guiding collaboration: Lessons learned in computer- 
supported collaboration learning research at Georgia Tech. Proc. Computer-Support for Collaborative Learning (CSCL '97), Toronto, Ontario, pp. 91-100.

Häkkinen, P., Järvelä, S. \& Mäkitalo, K. (2003). Sharing perspectives in virtual interaction: Review of methods of analysis. In Wasson, Ludvigsen \& Hoppe (2003), op. cit. (pp. 395404).

Hansen, T., Dirckinck-Holmfeld, L. Lewis, R. \& Rugelj, J. (2004). Using telematics for collaborative knowledge construction. In Dillenbourg (Ed.) (2004), op cit. (pp. 169-196).

Hermann, D. (Ed.) (2003). Narrative Theory and the Cognitive Sciences. Stanford, CA: Center for the Study of Language and Information.

Hollan, J., Hutchins, E., \& Kirsh, D. (2002). Distributed Cognition: Toward a New Foundation for Human-Computer Interaction Research. In J. M. Carroll (Ed.), Human-Computer Interaction in the New Millennium. (pp. 75-94). New York: ACM Press Addison Wesley. (Reprinted from ACM Transactions on Computer-Human Interaction, 7(2), June 2000.)

Hollan, J. \& Stornetta, S. (1992). Beyond being there. Proc. SIGCHI Conference on Human Factors in Computing Systems (CHI'92), May 3-7, 1992, Monterey, California, pp. 119125.

Jermann, P. \& Dillenbourg, P. (2003). Elaborating new arguments through a CSCL script. In Andriessen, Baker \& Suthers (2003), op cit. (pp. 205-226).

Jermann, P., Soller, A., \& Lesgold, A. (2004). Computer software support for CSCL. In Strijbos, Kirschner \& Martens (2004). op cit. (pp. 141-166).

Johnson, R. B \& Onwuegbuzie, A. J. (2004). Mixed methods research: A research paradigm whose time has come. Educational Researcher, 33(7), 14-26.

Jordan, B. \& Henderson, A. (1995). Interaction analysis: Foundations and practice. Journal of the Learning Sciences, 4(1), 39-103.

Kaput, J. \& Hegedus, S. (2002). Exploiting classroom connectivity by aggregating student constructions to create new learning opportunities In A. D. Cockburn \& E. Nardi (Eds.), Proceedings of the 26th Annual Conference of the International Group for the Psychology of Mathematics Education, Vol. 3. (pp. 177-184). University of East Anglia: Norwich, UK.

Kato, H., K. Yamazaki, K., Suzuki, H., Kuzuoka, H., Miki, H. \& Yamazaki, A. (2001). Designing a Video-Mediated Collaboration System Based on a Body Metaphor. In T. Koschmann, R. Hall \& N. Miyake (Eds.), CSCL 2: Carrying Forward the Conversation. (pp. 409-423). Mahwah, NJ, Lawrence Erlbaum.

Kirschner, P. A., Martens, R. L., \& Strijbos, J. W. (2004). CSCL in Higher Education? A Framework for Designing Multiple Collaborative Environments. In Strijbos, Kirschner \& Martens (2004). op cit. (pp. 3-30).

Koschmann, T. (2002). Dewey's contribution to the foundations of CSCL research. Proc. Computer Supported Collaborative Learning 2002, Boulder, January 7-11, 2002, pp. 1722.

Koschmann, T., \& LeBaron, C. (2003). Reconsidering common ground: Examining Clark's contribution theory in the OR. In K. Kuutti, E. Karsten, G. Fitzpatrick, P. Dourish, \& K. Schmidt (Eds.), ECSCW 2003: Proc. Eighth European Conference on Computer-Supported Cooperative Work. Amsterdam: Kluwer. 
Koschmann, T., Zemel, A., \& Stahl, G. (2004). The video analyst's manifesto (or the implications of Garfinkel's policies for the development of a program of video analytic research within the learning sciences). In Y. Kafai, W. Sandoval, N. Enyedy, A. Nixon, \& F. Herrera, Proceedings of the Sixth International Conference of the Learning Sciences (pp. 278-285). Mahwah, NJ: Lawrence Erlbaum Associates.

Koschmann, T., Zemel, A., Conlee-Stevens, M., Young, N., Robbs, J., \& Barnhart, A. (2003). Problematizing the problem: A single case analysis in a dPBL Meeting. In Wasson, Ludvigsen \& Hoppe (2003), op. cit., pp. 37-46.

Koschmann, T., Zemel, A., Conlee-Stevens, M., Young, N., Robbs, J. \& Barnhart, A. (2005). How do people learn? Members' methods and communicative mediation. In R. Bromme, F.W. Hesse \& H. Spada (2005), op. cit. (pp. 265-294.)

Kreijns, K. \& Kirschner, P. A. (2004). Designing sociable CSCL environments. In Strijbos, Kirschner \& Martens (2004). op cit. (pp. 221-243).

Latour, B. (1990). Drawing Things Together. In Lynch, M. \& Woolgar, S. (eds.) Representation in Scientific Practice. (pp. 19-68.) Cambridge, MA: MIT Press

Lave, J. \& Wenger, E. (1991). Situated Learning: Legitimate Peripheral Participation. Cambridge: Cambridge University Press.

Lingnau, A., Hoppe, H.U., Mannhaupt, G. (2003). Computer supported collaborative writing in an early learning classroom. Journal of Computer Assisted Learning, 19, 2, 186-194.

Matusov, E. (1996): Intersubjectivity Without Agreement. Mind, Culture and Activity. 3(1). 2545.

Norman, D. N. (1999). Affordance, conventions, and design. ACM interactions, 6(3), 38-43.

Nunberg, G. (1993). Indexicality and deixis. Linguistics and Philosophy, 16(1), 1993

Olson, G. M \& Olson, J. S. (2000). Distance Matters. Human-Computer Interaction, 15(2/3), September 2000. Reprinted in J. M Carroll (Ed) Human-Computer Interaction in the New Millennum. (pp. 397-417). New York: ACM Press (2002).

Pfister, H.R. (2005). How to support synchronous net-based learning discourses: Principles and perspectives. In R. Bromme, F.W. Hesse \& H. Spada (2005), op. cit.

Piaget, J. (1976). The Grasp of Consciousness: Action and Concept in the Young Child. Cambridge, MA: Harvard University Press.

Resnick, P. (2002). Beyond bowling together: Sociotechnical capital. In J. M. Carroll (Ed.), Human-Computer Interaction in the New Millennium. (pp. 647-672). New York: ACM Press Addison Wesley.

Rieber, L.P. (2004). Microworlds. In D. Jonassen (Ed.), Handbook of Research for Educational Communications and Technology (2nd ed.). (pp. 583-603). Mahwah, NJ: Lawrence Erlbaum Associates.

Robertson, J, Good, J., Pain, H. (1998). BetterBlether: The design and evaluation of discussion tool for education. International Journal of Artificial Intelligence in Education, 9. Online: http://cbl.leeds.ac.uk/ijaied/abstracts/Vol_9/robertson.html

Rogoff, B. (1995). Observing sociocultural activity on three planes: participatory appropriation, guided participation, and apprenticeship. In J. V. Wertsch, P. Del Rio, \& A. Alvarez (Eds.) Sociocultural Studies of Mind. (pp. 139-164.) New York: Cambridge University Press. 
Roschelle, J. (1994, May). Designing for cognitive communication: Epistemic fidelity or mediating collaborative inquiry? The Arachnet Electronic Journal of Virtual Culture, 2(2).

Roschelle, J. \& Teasley, S. D. (1995). The construction of shared knowledge in collaborative problem solving. In C. E. O’Malley (Ed.), Computer Supported Collaborative Learning (pp. 69-197). Berlin: Springer-Verlag.

Rummel, N., \& Spada, H. (2005). Sustainable support for computer-mediated collaboration. How to achieve and how to assess it. In R. Bromme, F.W. Hesse \& H. Spada (2005), op. cit.

Sacks, H., Schegloff, E. A., \& Jefferson, G. (1974). A simplest systematics for the organization of turn-taking in conversation. Language, 50(4), 696-735.

Salomon, G. (Ed.) (1993), Distributed Cognitions: Psychological and Educational Considerations. Cambridge: Cambridge University Press.

Scardamalia, M. \& Bereiter, C. (1991). Higher levels of agency for children in knowledge building: a challenge for the design of new knowledge media. The Journal of the Learning Sciences, 1(1), 37-68.

Schaffer, D. W. \& Clinton, K. A. (2005). Why all CSL is CL: Distributed mind and the future of computer supported collaborative learning. In T. Koschmann, D. Suthers \& T.W. Chan (Eds.) Computer Supported Collaborative Learning 2005: The Next 10 Years! (pp. 592601.) Mahwah, NJ: Lawrence Erlbaum Associates.

Shulman, L. S. (1987). Knowledge and teaching: Foundations of the new reform. Harvard Educational Review, 57, 1-22.

Slavin, R. E. (1995). Cooperative Learning: Theory, Research and Practice. (2nd ed.) Boston: Allyn \& Bacon.

Smolka, A. L. B, De Goes, M. C. R., \& Pino, A. (1995). The constitution of the subject: a persistent question. In J. V. Wertsch, P. Del Rio, \& A. Alvarez (Eds.) Sociocultural Studies of Mind. (pp. 165-184.) New York: Cambridge University Press.

Stahl, G. (2000) Collaborative information environments to support knowledge construction by communities, $A I \&$ Society, 14, 71-97.

Stahl, G. (2004). Building collaborative knowing: Elements of a social theory of CSCL. In Strijbos, Kirschner \& Martens (2004). op cit. (pp. 53-86).

Stahl, G. (in press). Group Cognition: Computer Support for Building Collaborative Knowledge. Cambridge, MA: MIT Press.

Star, S. L. (1990). The structure of ill-structured solutions: boundary objects and heterogeneous distributed problem solving. In L. Gasser and M. N. Huhns (Eds.) Distributed Artificial Intelligence: Vol. 2. (pp. 37-54). San Francisco: Morgan Kaufmann.

Strijbos, J .W., Kirschner, P. A. \& Martens, R. L. (Eds.) (2004). What We Know About CSCL and Implementing it in Higher Education. Dordrecht: Kluwer.

Suthers, D. (2005). Collaborative Knowledge Building through Shared Representations. Proc. 38th Hawai $i$ International Conference on the System Sciences (HICSS-37), January 3-6, 2005, Wakoloa, Hawai i (CD-ROM), Institute of Electrical and Electronics Engineers, Inc. (IEEE).

Suthers, D., Girardeau, L. \& Hundhausen, C. (2003). Deictic Roles of External Representations in Face-to-face and Online Collaboration. In Wasson, Ludvigsen \& Hoppe (2003), op. cit., pp. 173-182. 
Suthers, D., \& Hundhausen, C. (2003). An Empirical Study of the Effects of Representational Guidance on Collaborative Learning. Journal of the Learning Sciences, 12(2), 183-219.

Sweller, J., van Merriënboer, J. J. G., \& Paas, F. G. W. C. (1998). Cognitive architecture and instructional design. Educational Psychology Review, 10(3), 251-296.

Toth, E., Suthers, D., \& Lesgold, A. (2002). Mapping to know: The effects of evidence maps and reflective assessment on scientific inquiry skills. Science Education, 86(2), 264-286.

Van Der Pol, J., Admiraal, W., \& Simons, R-J. (2003). Grounding in electronic discussions: Standard (threaded) versus anchored discussion. In Wasson, Ludvigsen, \& Hoppe (2003), op. cit., pp. 77-81.

Von Glasersfeld, E., (1995). A constructivist approach to teaching. In L. Steffe \& J. Gale (Eds.). Constructivism in Education. (pp. 3-16). New Jersey: Lawrence Erlbaum Associates, Inc.

Vygotsky, L. S. (1978). Mind in Society: The Development of Higher Psychological Processes. Cambridge, MA: Harvard University Press. (Originally published in 1930.)

Wartofsky, M. (1979). Models, representation and scientific understanding. Boston: Reidel.

Wasson, B., Ludvigsen, S., \& Hoppe, U. (Eds), Designing for Change in Networked Learning Environments: Proc. International Conference on Computer Support for Collaborative Learning 2003. Dordrecht: Kluwer Academic Publishers.

Weinberger, A., Reiserer, M., Ertl, B., Fischer, F. \& Mandl, H. (2005). Facilitating collaborative knowledge construction in computer-mediated learning environments with cooperation scripts. In R. Bromme, F.W. Hesse \& H. Spada (2005), op. cit.

Wenger, E. (1987). Artificial Intelligence and Tutoring Systems: Computational and Cognitive Approaches to the Communication of Knowledge. Los Altos, Morgan Kaufmann.

Wenger, E. (1998). Communities of practice: Learning, meaning, and identity. Cambridge: Cambridge University Press.

Wenger, E., McDermott, R, Snyder, W. (2002). Cultivating Communities of Practice: a Guide to Managing Knowledge. Boston: Harvard Business School Press.

Whitworth, B., Gallupe, B., McQueen, R. (2000). A cognitive three-process model of computermediated group interaction. Group Decision and Negotiation, 9, 431-456.

Yukawa, J. (2005). Hearts and Minds through Hands Online: A Narrative Analysis of Online Learning through Co-Reflection in an Online Action Research Course. Unpublished doctoral dissertation, University of Hawai $i$. 